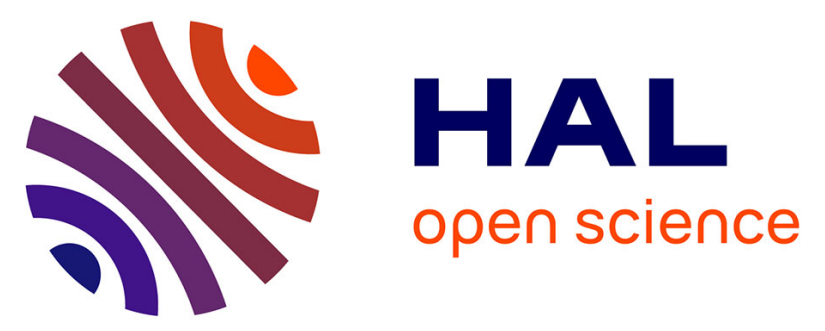

\title{
Pulse-Shape Analysis and position resolution in highly segmented HPGe AGATA detectors
}

\author{
L. Lewandowski, P. Reiter, B. Birkenbach, B. Bruyneel, E. Clement, J. \\ Eberth, H. Hess, C. Michelagnoli, H. Li, R.M. Perez-Vidal, et al.
}

\section{- To cite this version:}

L. Lewandowski, P. Reiter, B. Birkenbach, B. Bruyneel, E. Clement, et al.. Pulse-Shape Analysis and position resolution in highly segmented HPGe AGATA detectors. Eur.Phys.J.A, 2019, 55 (5), pp.81. 10.1140/epja/i2019-12752-0 . hal-02144357

\section{HAL Id: hal-02144357 \\ https://hal.science/hal-02144357}

Submitted on 13 Nov 2020

HAL is a multi-disciplinary open access archive for the deposit and dissemination of scientific research documents, whether they are published or not. The documents may come from teaching and research institutions in France or abroad, or from public or private research centers.
L'archive ouverte pluridisciplinaire HAL, est destinée au dépôt et à la diffusion de documents scientifiques de niveau recherche, publiés ou non, émanant des établissements d'enseignement et de recherche français ou étrangers, des laboratoires publics ou privés. 


\title{
Pulse-Shape Analysis and position resolution in highly segmented HPGe AGATA detectors
}

\author{
L. Lewandowski ${ }^{1}$, P. Reiter ${ }^{1}$, B. Birkenbach ${ }^{1}$, B. Bruyneel ${ }^{1}$, E. Clement ${ }^{2}$, J. Eberth $^{1}$, H. Hess ${ }^{1}$, C. Michelagnoli ${ }^{2}$, R.M. \\ Perez-Vidal $^{3}$, and M. Zielinska ${ }^{4}$ \\ 1 University of Cologne, Institut für Kernphysik, Zülpicher Strasse 77, 50937 Köln, Germany \\ 2 Grand Accélérateur National d'Ions Lourds, Boulevard Henri Becquerel, 14000 Caen, France \\ 3 Instituto de Fisica Corpuscular, CSIC-Universitat de Valencia, E-46920 Valencia, Spain \\ 4 CEA Saclay, Service de Physique Nucleaire, 91191 Gif-sur-Yvette, France
}

Received: date / Revised version: date

\begin{abstract}
The performance of the Pulse-Shape Analysis (PSA) in AGATA HPGe detectors was investigated and improved employing a $\gamma$-ray source measurement based on $e^{-} e^{+}$annihilation radiation after decays of ${ }^{22} \mathrm{Na}$ by $\beta^{+}$decay. The first interaction positions of the two $511 \mathrm{keV} \gamma$ rays were determined and the connecting line of these two positions was compared to the known source position as a measure for the PSA performance. The position resolution and its dependence on the PSA parameters were investigated by varying most relevant input quantities: the charge carrier mobility of the holes, the response of the employed measuring electronics especially the preamplifier rise time. The relative statistical weight of charge signals and transient signals was scrutinized. The optimal distance metric of the grid-search algorithm and its impact on the position resolution was determined.
\end{abstract}

PACS. 29.30.Kv X- and $\gamma$-ray spectroscopy - 29.40.Gx Tracking and position-sensitive detectors -29.40 .Wk Solid-state detectors - 07.05.Tp Computer modeling and simulation

\section{Introduction}

AGATA in its final configuration will be a highly efficient $4 \pi \gamma$-ray spectrometer consisting of 180 highly segmented HPGe detectors [1]. It will provide utmost detection efficiency and performance employing the $\gamma$-ray tracking (GRT) technique which is based on the individual interaction positions of all $\gamma$ rays within the segmented HPGe detector volumes of the array [2]. The interaction positions of the $\gamma$ rays within the individual segments of the HPGe detectors are obtained by means of Pulse-Shape Analysis (PSA) using the adaptive grid search [3]. The main idea of PSA with highly segmented HPGe detectors is a detailed comparison between the measured detector pulses and a set of reference pulse shapes which are generated for a narrow three dimensional grid in order to identify the most probable interaction position within the active detector material. These reference pulse shapes comprise information created by moving charge carriers and influenced signals. The pulse shapes unambiguously depend on the three dimensional location of the interaction point where electron hole pairs are created after the interaction of the incoming $\gamma$ ray. The position information of the grid point together with the pulse shape information of the core and the segment signals are bundled in lookup libraries. To achieve the necessary position resolution of a few millimeters the calculated signal pulses from different interaction points have to be equal or very similar to the measured pulses.

The theoretical signal pulses are extracted from simulations which request very accurate information on the properties of each individual detector. The procedure how these position dependent detector pulses are generated for the AGATA detectors is given by the AGATA Detector Library (ADL) described in Ref. [4]. Each individual HPGe detector is characterized by its geometry, space-charge distribution, crystal-axis orientation, crosstalk properties and the response function of the electronics. The interaction positions allocated by the PSA depend also on the grid-search algorithm that is employed to perform the comparison of the measured and simulated pulses. Moreover, the results of this comparison depend on the time alignment between measured and calculated pulses, the distance metric and the weighting of the individual segment pulses.

Recent studies demonstrated that the AGATA PSA can resolve the interaction position of $\gamma$ rays in segmented AGATA detectors with an energy dependent position resolution of a few millimeters [5,6]. The AGATA tracking efficiency is also within the specifications. However, an inspection of the interaction position yielded for example an unexpected clustering of position points as a result of the PSA, which are not in agreement with the expected distribution of hits within the Ge detector volume. Especially, measurements with a ${ }^{60} \mathrm{Co}$ source show deviations from 
isotropic distributions and a clustering of hits in specific parts of the detector volume or even a clear surplus of hits at single grid points (see chapter 3 ) is observed. The clustering of positions and accumulation of grid points in the final results of the AGATA PSA inspired a refined investigation of the AGATA PSA.

In the first part of this paper a reminder to the principles of the AGATA PSA will be given and results from latest source measurements will be discussed. The second part covers the coincident detection of $511 \mathrm{keV} \gamma$ rays at $180^{\circ}$ and the reconstructed emission point. Its comparison to the known source position enables a detailed assessment of the PSA performance. In the third part of the paper input parameters are varied and optimized by maximizing the position resolution of the PSA.

\section{PSA principles}

The Pulse-Shape Analysis combines the measured digitized traces of AGATA detector pulses from individual HPGe detectors and the AGATA data library (ADL) employing the adaptive grid search $[3,4]$ algorithm. The measured detector signals from individual $\gamma$-ray events are taken from the 36 segment signals and the core signal from the analogue preamplifiers after $100 \mathrm{MHz}$ digitization or $10 \mathrm{~ns}$ time steps with the AGATA electronics (see also Ref. [1]). The ADL contains simulated pulses of every segment and the central electrode (core) for every possible interaction position in the detector. The detector is divided into a grid with a set of simulated signals associated with every grid point. All the pulses from the position dependent simulation are compared to the measured detector pulses. The set of pulses with the best matching of measured and simulated pulses is used to assign the interaction position of the $\gamma$ ray.

The adaptive grid search yields first an interaction position which is the result of a three dimensional search in a coarse grid with a length of 6 millimeters per grid point. Then a refined second search is conducted around the interaction position found in the coarse search. The fine grid has a length of 2 millimeters per grid point. The comparison of measured and simulated signals is done by minimizing the following figure of merit:

$$
\text { Figure of Merit }=\sum_{j, t_{i}}\left|A_{j, m}\left(t_{i}\right)-A_{j, s}\left(t_{i}\right)\right|^{p}
$$

$A_{j, m}\left(t_{i}\right)$ and $A_{j, s}\left(t_{i}\right)$ are the pulse heights at the time $t_{i}$ of segment $j$ of the measured $(m)$ or simulated $(s)$ pulse. Only the hit segment, its nearest neighbors and the core signal are considered. Typically, a time range of the first $400 \mathrm{~ns}$ or 40 time steps after the determined $T_{0}$ are used to determine the figure of merit. The exponent $p$ is a real number greater zero that has to be chosen. The adaptive grid search provides only one interaction per detector segment.

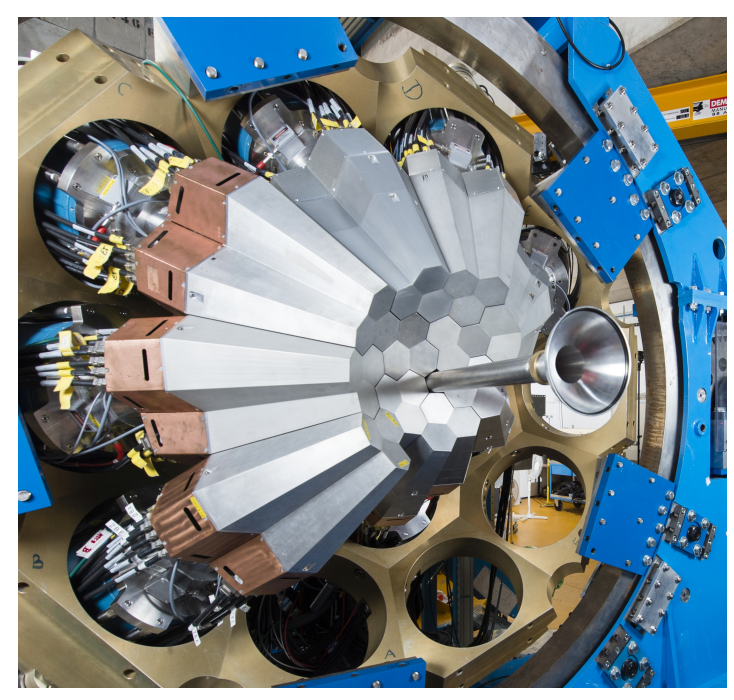

Figure 1. Picture of the AGATA array at GANIL which comprised 29 individual HPGe detectors at this time. The crystals have a tapered hexagonal geometry with an asymmetric shape to fit into a $4 \pi$ configuration with a 180 detector geometry. A beam pipe and an open target chamber is located in the central position of the setup. A ${ }^{60} \mathrm{Co}$ source was placed in the middle of the vacuum chamber for calibration. ${ }^{1}$

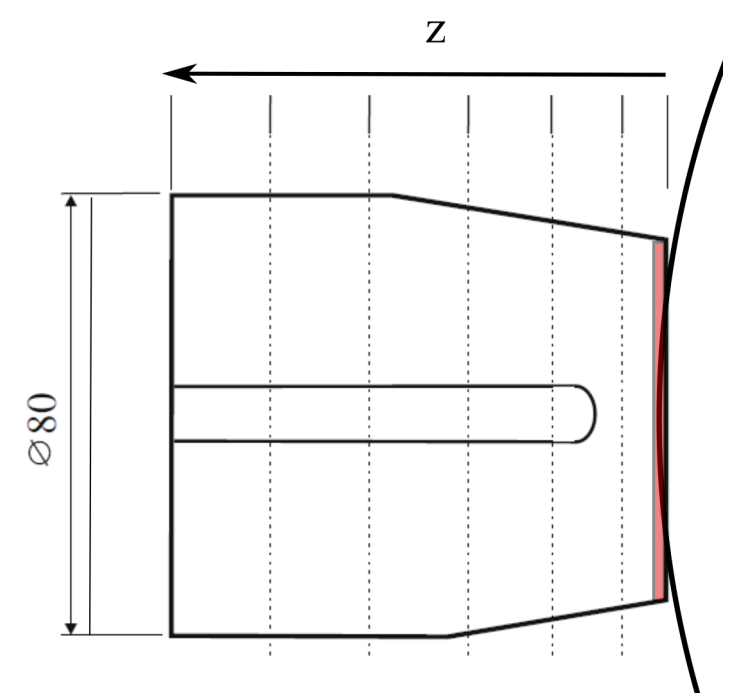

Figure 2. Schematic side view of the AGATA HPGe detector. The black sector of a circle indicates the positions with the same distance to the source. Sets of grid points at a constant detector depth $z$ have approximately the same distance to the source. This is indicated for the front part at $z=0-2 \mathrm{~mm}$ (red marked area). A maximum deviation to the sphere is about $2.5 \mathrm{~mm}$ compared to the smallest radial distance of $226 \mathrm{~mm}$.

\section{Results of the Pulse-Shape Analysis}

A measurement with a ${ }^{60} \mathrm{Co}$ source was performed at the Grand Accélérateur National d'Ions Lourds (GANIL) with 29 HPGe detectors comprised in nine triple-cluster and one double-cluster detectors. The source was located in

\footnotetext{
1 Picture by P. Stroppa, CEA
} 

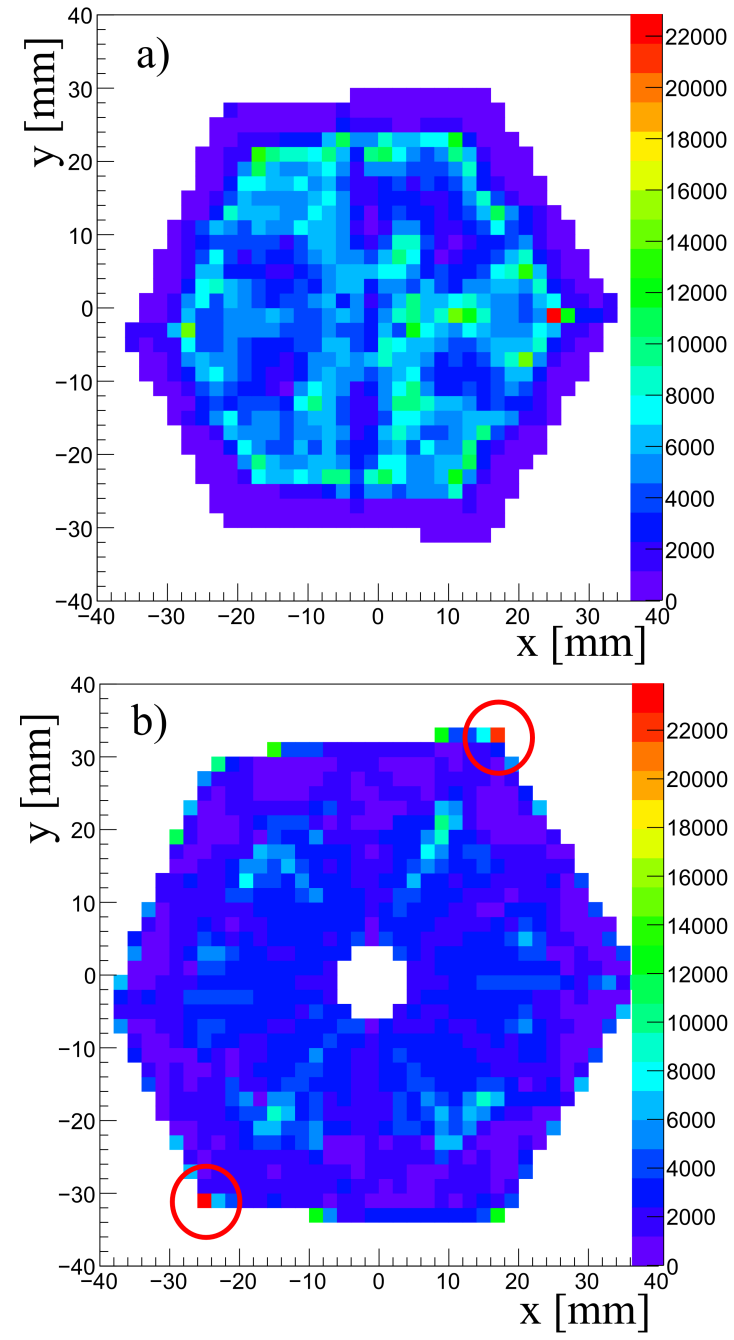

Figure 3. The distribution of hits allocated by the PSA is shown for crystal A001 for two different depth values of a) $z=6-8 \mathrm{~mm}$ and b) $z=28-30 \mathrm{~mm}$. The first picture a) shows a reduced number of hits for large radii. In addition the six fold structure of the segments is visible. The second picture b) illustrates that the PSA assigns a much higher amount of hits in single grid points at the upper right and lower left corners marked with circles. For both pictures a homogeneous distribution of hits with statistical fluctuation is expected.

the center of the sphere at a distance of $226 \mathrm{~mm}$. The data set was analyzed using the adaptive grid search and the AGATA data library introduced in section 2. For a given distance $z$ along the main axis of the HPGe detector each grid point has approximately the same distance to the source. The distance deviates by approximately $1 \%$ for different points of that plane located at the fringes of the detector volume (see Fig. 2). Therefore, the expected number of hits for a detector plane with constant $z$-value is estimated to be equal within statistical errors for a source measurement.

However, the interaction positions allocated by the PSA results show deviations from these expectations which cannot be explained by the statistical nature of the mea-

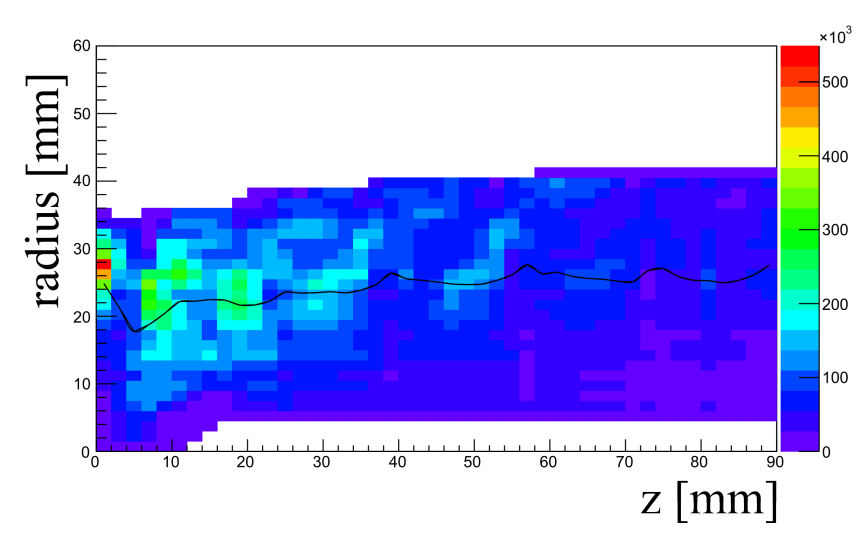

Figure 4. The radii of the interaction positions are plotted versus the detector depth $z$ for crystal A001. The black line marks the mean radius for the given depth $z$. The source is placed in front of the crystal at a distance of $226 \mathrm{~mm}$. A clustering of hits is observed at specific depth values $z$ which is not expected illustrating a systematic deviation between the PSA results and the physical interaction position.

surement. The six fold structure of the segments is visible although the interaction probability should not be impacted by the segmentation (for more details on the segment structure refer to [1]). Some grid points at the outer edges of the detector or in the middle of segments show more hits than expected (see Fig. 3). The deviations from the smooth distribution indicate that some of the allocated interaction positions systematically disagree with the physical interaction points (see also Fig. 4).

The pulses that are created by the AGATA Data Library depend on a variety of detector properties and the acquisition electronics. For the calculation several input parameters for each individual HPGe detector are needed to generate the most reliable set of pulse shapes for the ADL. Most of these input parameters are measured routinely, e.g. the cross talk properties of the detectors of its electronics network. However, some quantities, like the space charge distribution inside the Ge crystal volume, are hard to access and are only partially available from the crystal manufacturer.

In order to understand the clustering of interaction points and to optimize the PSA performance a method was developed that scrutinizes the PSA input parameters within meaningful ranges especially in case the parameters are not measurable or known with uncertainties. It will be introduced in the following section 4 .

\section{Coincidence measurement of $\gamma$ rays after $e^{+} e^{-}$annihilation}

The position sensitivity of several AGATA detectors was determined by analyzing the data of a ${ }^{22} \mathrm{Na}$ source measurement. In the decay process of ${ }^{22} \mathrm{Na}$ a positron is emitted. This positron annihilates with an electron very close to the point of emission. It is assumed that the source is point like and that the positron annihilates at the point of emission. 


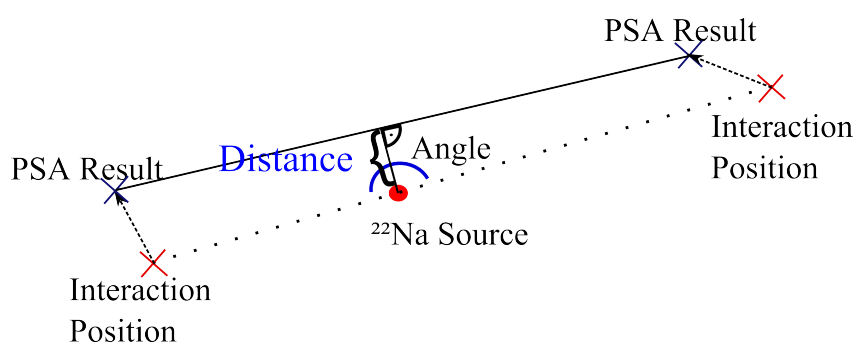

Figure 5. Two $511 \mathrm{keV} \gamma$ rays are emitted after $e^{+} e^{-}$annihilation and both are detected in coincidence in opposite Ge crystals. The connecting line of the two interaction positions found by the PSA may not cross the known source position. This is mainly caused by the finite position resolution of the PSA and to a minor part by the range of the positron. The minimum distance of the connecting line to the source position provides a measure for the performance of the PSA.

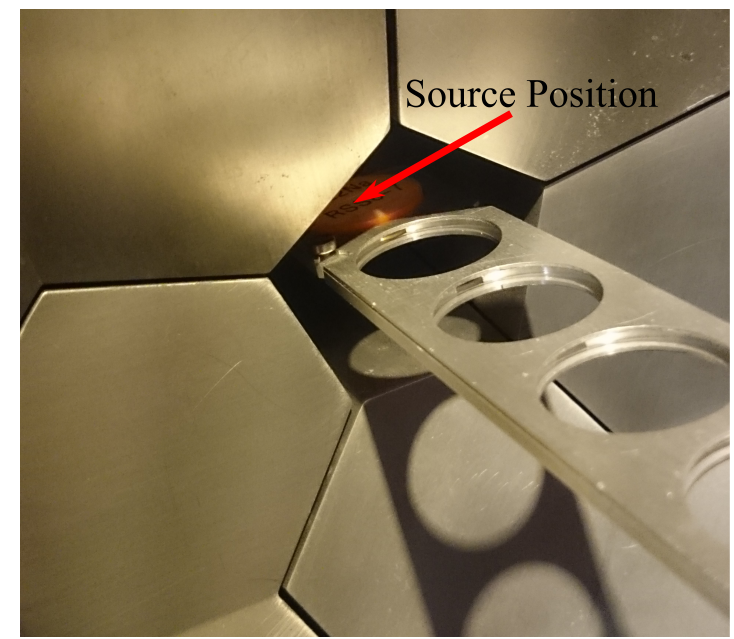

Figure 6. The source was placed $3.2 \mathrm{~cm}$ inside the pentagonal shaped hole which is typically used for the beam line. This geometry allows the coincident detection of the $180^{\circ}$ correlation of the two $\gamma$ rays for several crystals and ensures that data is also available for the segments located at the back side of the crystals.

The positron emitted by ${ }^{22} \mathrm{Na}$ has a maximum energy of $540 \mathrm{keV}$ and a mean energy of $180 \mathrm{keV}$. The mean range of the positron in polyethylen is about $0.24 \mathrm{~mm}$ [7]. The assumption that two $511 \mathrm{keV} \gamma$ rays are emitted with an angle of $180^{\circ}$ is very well justified. The coincident detection of these $\gamma$ rays allows to assess the quality of the PSA results. For this purpose, the shortest distance of a straight connecting line between the two found interaction points to the known source position is determined (see Fig. 5 for details). A similar approach was also used in the past to compare different simulated signal bases by [8].

For the measurement the ${ }^{22} \mathrm{Na}$ source was positioned within one of the pentagonal shaped holes. It was placed $3.2 \mathrm{~cm}$ inside the spherical shell formed by the canisters of the triple cluster detectors close to the five surrounding individual HPGe detectors (see Fig. 6). Coincident events with an energy of $511 \pm 3 \mathrm{keV}$ were selected allowing only for two interactions in the complete array in two individ- ual crystals. The background of random coincidences was reduced by gating on angles larger than $150^{\circ}$ between two $\gamma$ rays positions and the source (see Fig. 9). Assuming a constant background of 250 counts $/{ }^{\circ}$ in the range of 150 $180^{\circ}$ only $1.53 \%$ of all events in that region are estimated to be from background.

Very large deviations from $180^{\circ}$ cannot be caused by the position uncertainty of the PSA. The distance from the connecting line of the measured interaction positions to the source position (see Fig. 5) was required to be shorter than $14 \mathrm{~mm}$. Events with larger distances were discarded since they are most likely caused by random coincidences.

The trigger rate for the five inner crystals was approximately $1 \mathrm{kHz}$ and $200 \mathrm{~Hz}$ for all other detectors. For the event building a timing window of $\pm 2.5 \mu$ s was chosen. Fig. 7 shows the time stamp differences of the individual detectors before and after applying the aforementioned data analysis conditions. With these gates, a data set of about $468 \mathrm{k}$ events was selected for the final analysis. The mean distance from the distribution of minimum distances between the source position and the connecting line (see Fig. 10), accumulated for all coincidences, was used as a measure for the quality of the PSA. In order to investigate and improve the PSA performance the input parameters were varied and optimized in a variation process.

The ${ }^{22}$ Na-coincidence method is used to verify properties of the crystal and the electronics as well as parameters of the algorithm. The position uncertainty of both interactions of different detectors contribute to the final performance measurement. Therefore, crystal specific properties, such as the impurity concentration of the individual crystals, are not subject of the following procedure.

\section{Improving PSA performance}

The results of the PSA rely on the detailed comparison of calculated detector signals which are generated by the AGATA data library. These signals depend on the individual HPGe crystal properties and the signal processing by analog and digital electronics. Moreover, the results depend on the adaptive grid search algorithm that compares measured and simulated traces. The ${ }^{22} \mathrm{Na}$-coincidence method introduced in section 4 is employed to optimize the characterization of the detector and electronics parameters as well as the grid search algorithm. A variation procedure is employed to re-evaluate and to improve the individual PSA parameters. For this purpose the input parameter of interest (e.g. a crystal property) was varied in a certain range around the previously used value. For each individual variation step the same ${ }^{22} \mathrm{Na}$-coincidence data set was analyzed again, leading to new interaction positions found by the PSA. This set of new interaction positions was then searched for $\gamma \gamma$ coincidences as described in section 4 . As a result of the variation procedure the local minimum of the mean distance of the lines to the source (see Fig. 8 and 10) was determined.

The reanalysis of the data set is CPU time consuming which is mainly caused by the grid search algorithm of the PSA. In addition the analyzed data set was chosen 

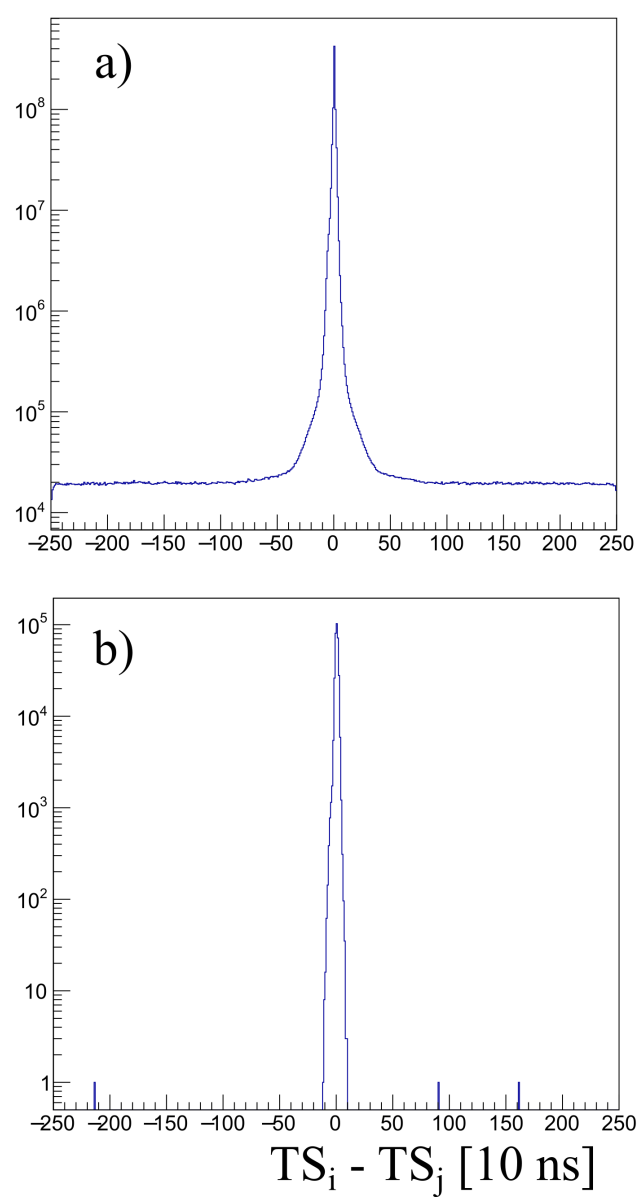

Figure 7. The differences of the time stamps $T S_{i, j}$ for all individual detector combinations $i$ and $j$ are plotted in a). In addition to correlated interactions close to zero a constant background is observed. The same difference is plotted in b) after applying gates to multiplicity, energy, angle and distance reducing the background nearly to zero (for details see text).

to be sufficiently large to ensure a high significance. The used data set consisted of $6.24 \cdot 10^{8}$ individual interactions leading to typical computing times of around a day for a single variation step using a $32 \mathrm{CPU}$ core high performance server. Therefore, variations were performed for single parameters because a multidimensional variation was not feasible due to time constraints. However, clearly correlated quantities were studied simultaneously like e.g. the hole velocity parameter and the preamplifier rise time.

\subsection{Transfer function}

The measured pulses are processed and shaped by the preamplifier and the digitizer electronics $[9,10]$. In order to compare the measured pulses with calculated signals the transfer function of the electronics is needed for the convolution of the the simulated signals with this transfer function. The properties of the transfer function is described in a simplified approach by a single rise time

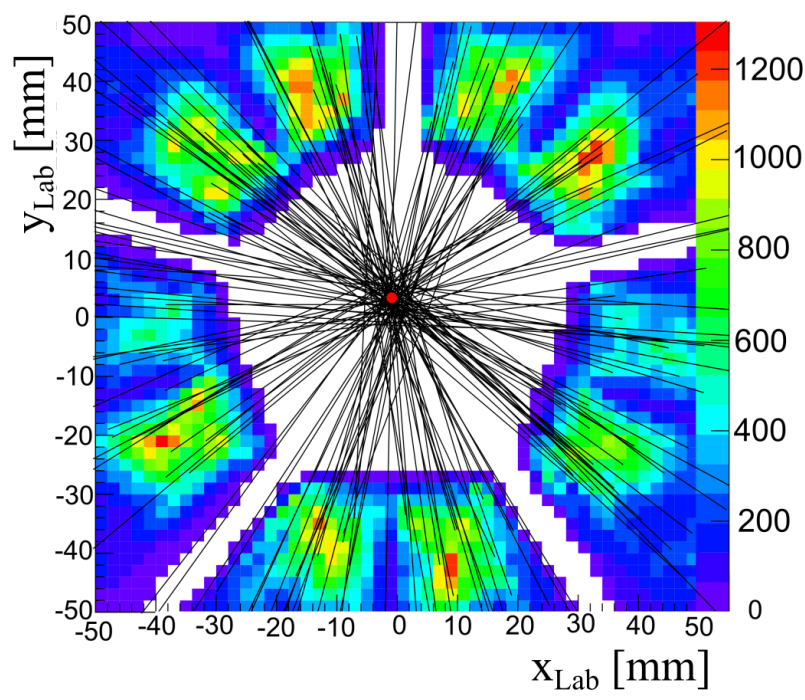

Figure 8. A visualization of the measured interaction positions of all $511 \mathrm{keV} \gamma \gamma$ coincidence events is shown (colored distribution). A line is drawn between the two interaction positions. These lines pass by in close distance to the source position which is marked with a red dot. For clarity only lines from 100 coincident events are plotted.

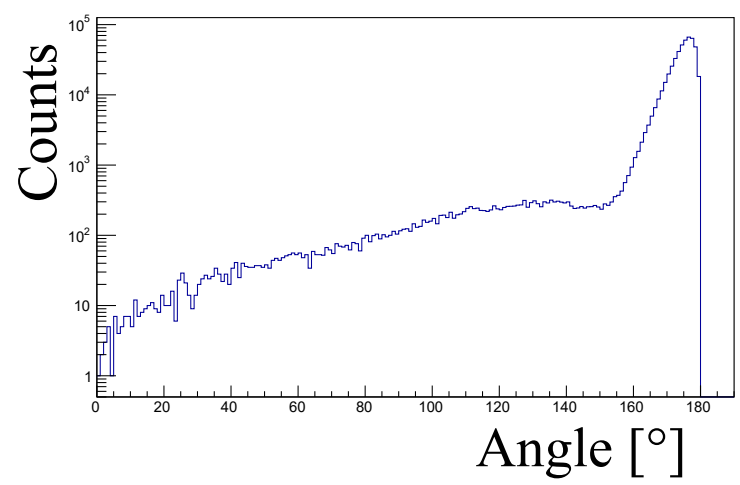

Figure 9. The distribution of angles between the two $511 \mathrm{keV}$ interactions is shown. For background reduction only events with an angle larger than $150^{\circ}$ were considered which corresponds to $96.56 \%$ of all events.

$\tau$ of the preamplifier. Consequently, this rise time was varied and the different results were evaluated with the method presented in section 4 . The optimal value for the position resolution given by the mean value is found to be around $45 \mathrm{~ns}$, (see results of Fig. 11), a value which is about $10 \mathrm{~ns}$ larger than expected from measurements of the preamplifier rise times [11]. This deviation is expected to be caused by an additional contribution to the measured signal rise time from the digitizers electronics. Another explanation for a slower measured rise time may also be given by the drift velocities of the charge carriers emplyed in the simulation. Therefore, the mobility of charge carriers and its interplay with the transfer function was investigated separately (see section 5.2). 


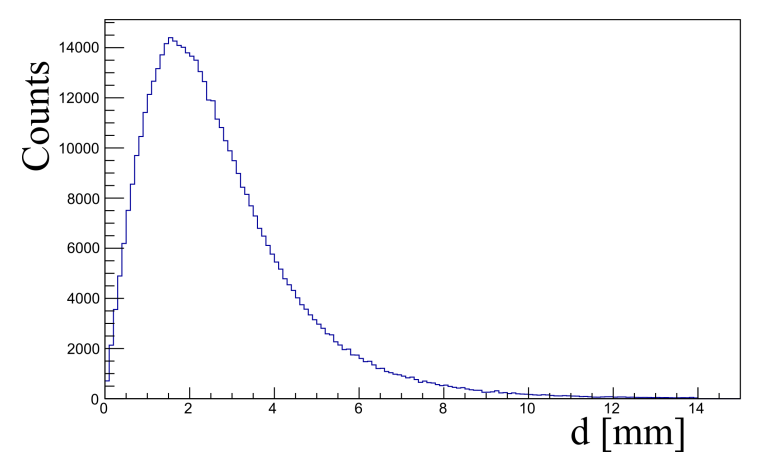

Figure 10. The distance of the connecting lines between found coincidences and the source position is shown. The mean of this distribution is used as a measure for the PSA performance. The standard AGATA PSA was used and a mean distance of $2.73 \mathrm{~mm}$ and a standard deviation of $1.85 \mathrm{~mm}$ was achieved.

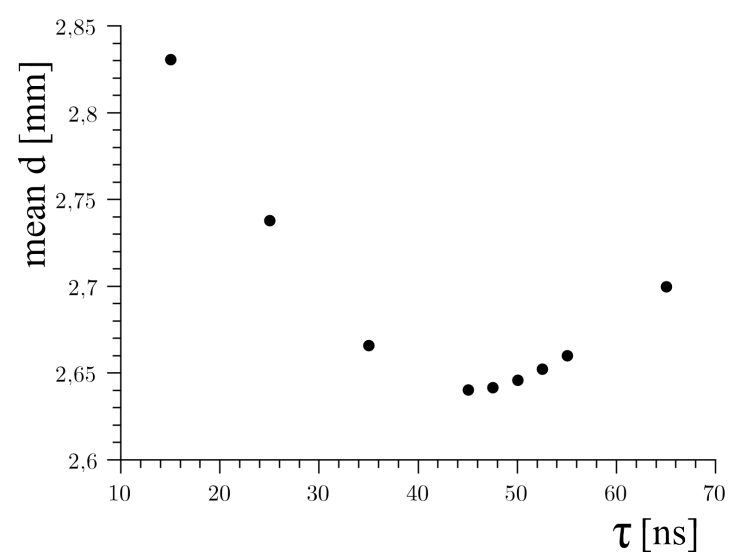

Figure 11. The rise time of the transfer function was varied in a wide range of $15-65 \mathrm{~ns}$ around the expected best value which is obtained at $\tau=45 \mathrm{~ns}$.

\subsection{Hole mobility}

The drift velocity of the charge carriers is essential for calculating precise pulses for the different interaction positions. For the hole drift velocity the empirical approach of $[12,13,14]$ is employed:

$$
v_{D}=\frac{\mu E}{\left(1+\left(\frac{E}{E_{0}}\right)^{\beta}\right)^{\frac{1}{\beta}}}
$$

Where $v_{D}$ is the drift velocity of the holes, $\mu$ the mobility of the holes and $E$ the electrical field strength. $E_{0}$ and $\beta$ are empirically determined parameters. The used values are given in table 1 which were derived by Bruyneel et al. in the same way as described in [11].

The hole mobility $\mu$ was varied in the range between 40 and $70 \frac{\mathrm{cm}^{2}}{\mathrm{mVs}}$. Due to the time consuming manner of the variation procedure the mobility was assumed to be the same in every crystallographic direction. This is a valid approximation as the mobility in the $\langle 100\rangle$ and $\langle 111\rangle$ directions only differ by less than one percent. The results in Fig. 12 show the best PSA performance for a hole
Table 1. The used values for the calculation of the hole drift velocities depends on the two different crystallographic directions $\langle 100\rangle$ and $\langle 111\rangle$ of the Ge crystal. Values are taken from [4]

\begin{tabular}{lll}
\hline & $\langle 100\rangle$ & $\langle 111\rangle$ \\
\hline$\mu\left[\frac{\mathrm{cm}^{2}}{m V^{s}}\right]$ & 62.934 & 62.383 \\
$E_{0}\left[\frac{V^{s}}{c m}\right]$ & 181.9 & 143.9 \\
$\beta$ & 0.73526 & 0.7488
\end{tabular}

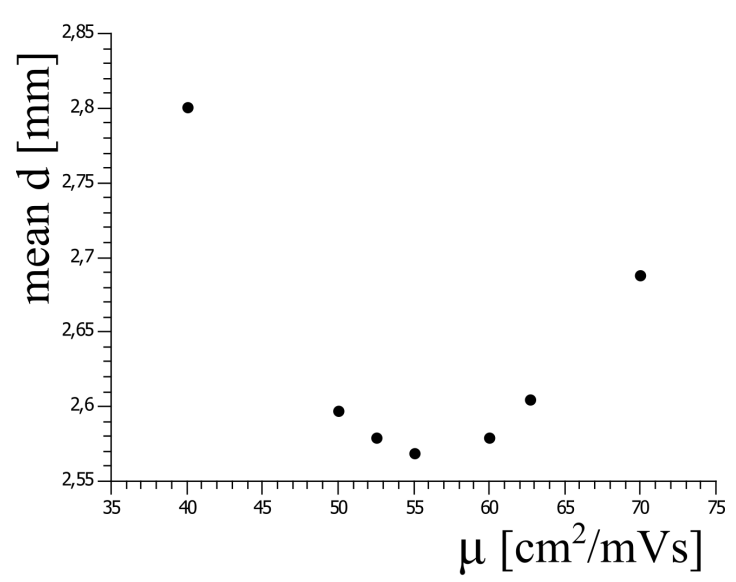

Figure 12. Variation of the hole mobility in germanium. To reduce the needed computational time the mobility for the $\langle 100\rangle$ and $\langle 111\rangle$ directions were chosen identically. The PSA performs best for a mobility of $\mu=55 \frac{\mathrm{cm}^{2}}{\mathrm{mVs}}$.

mobility of $\mu=55 \frac{\mathrm{cm}^{2}}{\mathrm{mVs}}$. The variation was performed with a rise time of $\tau=35 \mathrm{~ns}$. The results in section 5.1 show that a slower preamplifier response yields improved results. Using a larger preamplifier rise time has a very similar consequence on the simulated traces like using a reduced mobility. Therefore, both of these input parameters were investigated in combination by varying both in an iterative procedure. For this either the hole mobility $\mu$ or the rise time $\tau$ were fixed and the other parameter was varied.

Best results (in terms of a minimal mean distance $d$ ) were obtained for a rise time of $\tau=40 \mathrm{~ns}$ and $\mu=55 \frac{\mathrm{cm}^{2}}{\mathrm{mVs}^{2}}$. Note that the drift velocity also depends on several other factors as for example the impurity profile, the empirical parameters $E_{0}$ and $\beta$ as well as the electrical field strength.

\subsection{Weighting of transient signals}

The signals of the hit segment and the core are very sensitive to a change in the radius of the interaction position but change only slightly for different angles $\phi$ or different $\mathrm{z}$ values (see Fig. 15, the terms radius, $\mathrm{z}$ and $\phi$ are used as in a cylindrical coordinate system of the HPGe detector volume). The transient signals are essential for extracting correct $\phi$ angles and $\mathrm{z}$ values as they provide a very characteristic change of signal shapes as a function of these coordinates (see Fig. 13 and 14). 


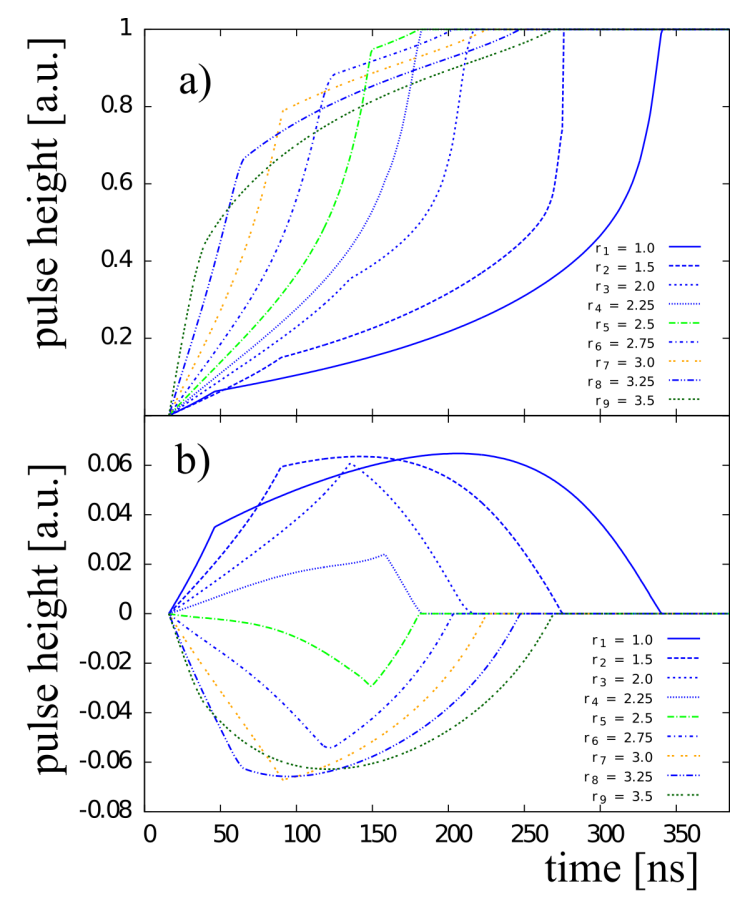

Figure 13. Simulated traces are shown for different radii at an fixed angle $\phi=15^{\circ}$ and a depth $z=40 \mathrm{~mm}$ for a) the hit segment and b) for the left neighbor. The pulse shapes of the hit segment are very sensitive to a change in the radius of the interaction. Even more, the transient signals in the neighboring segment also change the signal shape in a characteristic way for different radii. Here the polarity of the signal changes going from inner to outer radii and the induced signals nearly vanish for intermediate radii in the middle of the segment.

Therefore, the transient signals need to have a considerable impact on the final figure of merit although they are typically relatively small compared to the signals of hit segment and core. The figure of merit landscape for a single event is depicted in Fig. 15 illustrating the higher sensitivity to radial changes than to angular changes in the interaction position.

The contribution of the transient signals to the final figure of merit is in average about $35 \%$, depending on the choice of $p$ in eq. 1 and on the interaction position. A weighting was introduced to explore the contribution of the transient signals to the final figure of merit value and to increase the angular position resolution. The modified weighted figure of merit is given by:

$$
\text { Figure of Merit }=\sum_{j} w_{j} \sum_{t_{i}}\left|A_{j, m}\left(t_{i}\right)-A_{j, s}\left(t_{i}\right)\right|^{p}
$$

Here $w_{j}$ is a weighting coefficient that is one for the hit segment and the core. The weighting factor was determined for the nearest neighbors. Segments that are neither hit nor adjacent to a hit segment are not considered. The weighting coefficient was varied assessing the PSA performance for each variation step. The results are shown in Fig. 16 and an optimal value of about $w_{j}=2.75$ is obtained. The PSA yields better results by increasing the weighting coefficient

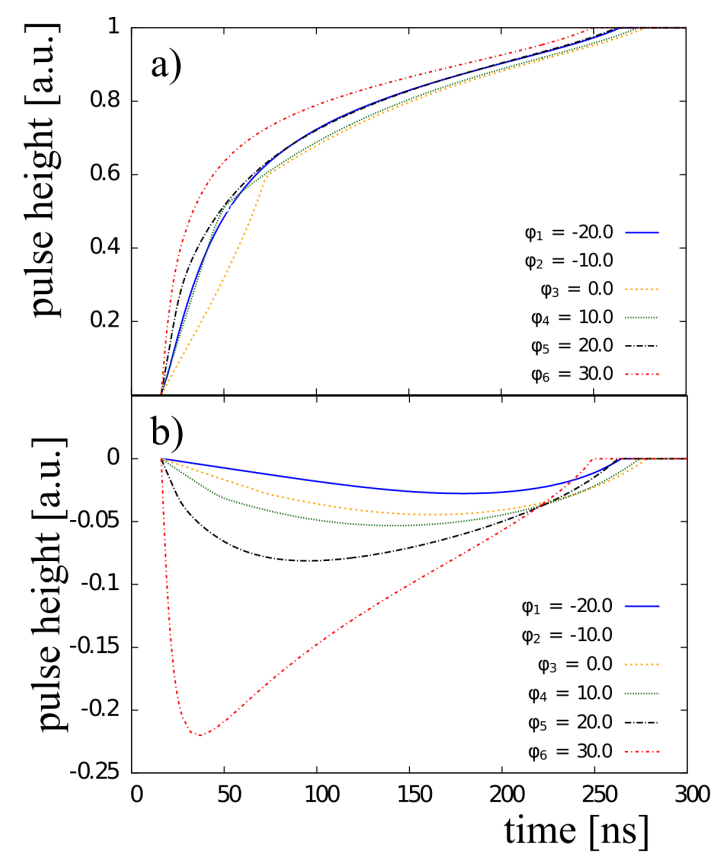

Figure 14. Simulated traces for different angles $\phi$ are shown for a fixed radius of $r=35 \mathrm{~mm}$ and a depth of $z=40 \mathrm{~mm}$ for a) the hit segment and b) for the left neighbor. The change in the pulse shape of the hit segment is relatively small whereas the transient signals provide a pronounced change in amplitude for different $\phi$ angles.

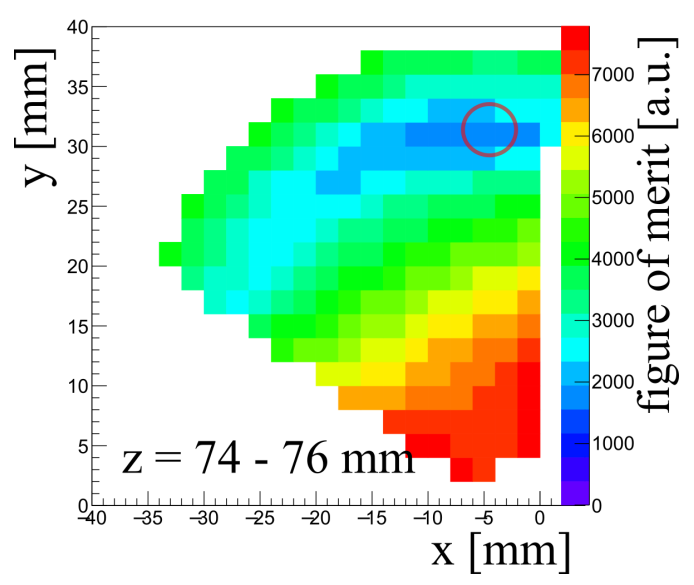

Figure 15. The figure of merit associated with every possible interaction position with a fixed $z$ is shown for a single event. The minimum, corresponding to the allocated interaction position, is marked with a red circle. The change of the figure of merit is much more pronounced in radial direction than for different angles $\phi$.

as the figure of merit is governed by the contribution of the transient signals in this case.

\subsection{Distance metric}

The calculation of the figure of merit (see eq. 1) depends as well on the choice of the exponent $p$, that determines 


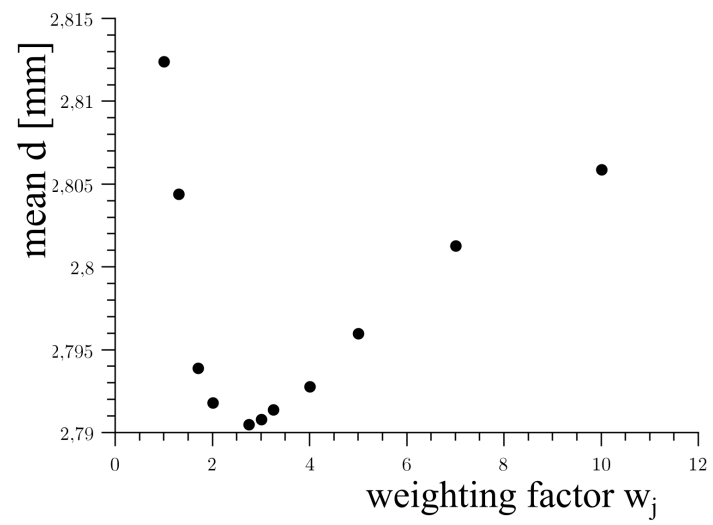

Figure 16. Variation of the weighting coefficient in the figure of merit calculation, see eq. 3. Better PSA performance was achieved with a weighting coefficient of about 2.75 compared to the standard non weighted comparison of measurement and simulation.

the distance metric (e.g. $p=2$ corresponds to an Euclidean metric which is used for standard $\chi^{2}$ minimization). The value of $p$ has considerable effect on the comparison between measured and simulated pulses and on the result which minimizes the figure of merit best. Large values of the exponent $p$ correspond to a stronger weighting of large deviations of measured and simulated pulses. In this way the impact of noise is reduced as these deviations are typically small. On the other hand, the transient signals are most relevant for the correct position determination by the PSA. Depending on the interaction position, these can be very small compared to the signals of the hit segment or of the core. Therefore, $p$ cannot be not too large.

To investigate this behaviour PSA was performed for different values of the exponent $p$. Each time the PSA result was assessed with the ${ }^{22} \mathrm{Na}$-coincidence method introduced in section 4 . The results are depicted in Fig. 17. The found minimum corresponds to a value of $p=0.4$ which shows that smaller values than the Euclidean metric is favored in this case. The result is consistent with previous findings where an optimal value of $p=0.3$ was found using the quality of the Doppler correction from in-beam data as performance criterium [15]. This also implies that the differences between simulation and measurement are not Gaussian like (see also Fig. 18).

An ideal scenario would be free of systematic errors; the deviations between measurement and simulation only arise from the noise of the signal and the difference of both is expected to be Gaussian. However, the observed differences of measurement and simulation cannot be solely explained by noise. Additional deviations are presumably caused by the non perfect data base of simulated signals and simplifying assumptions that are used in the grid-search algorithm which leads to systematic deviations between measurement and simulation, for example the simplifying assumption of only one interaction per segment.

As shown in section 5.3 a weighting of the transient signals is favorable. This implies that a different distance

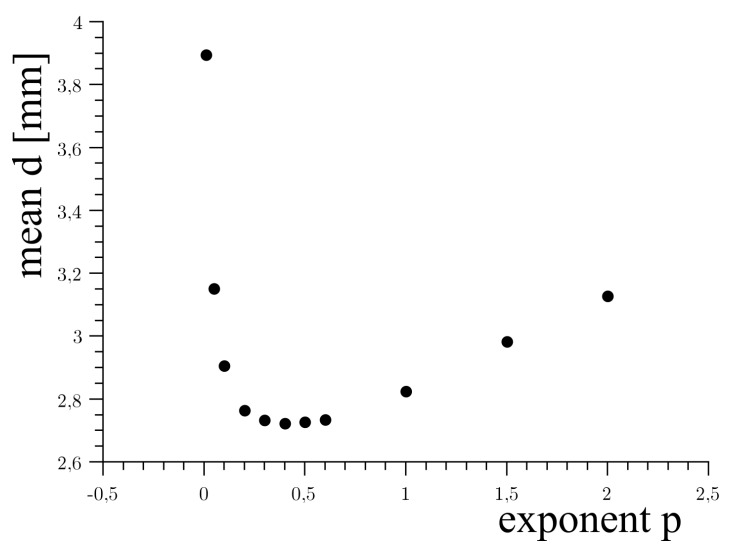

Figure 17. Variation of the exponent $p$ in the calculation of the figure of merit. The minimum at $p=0.4$ corresponds to the best PSA results.
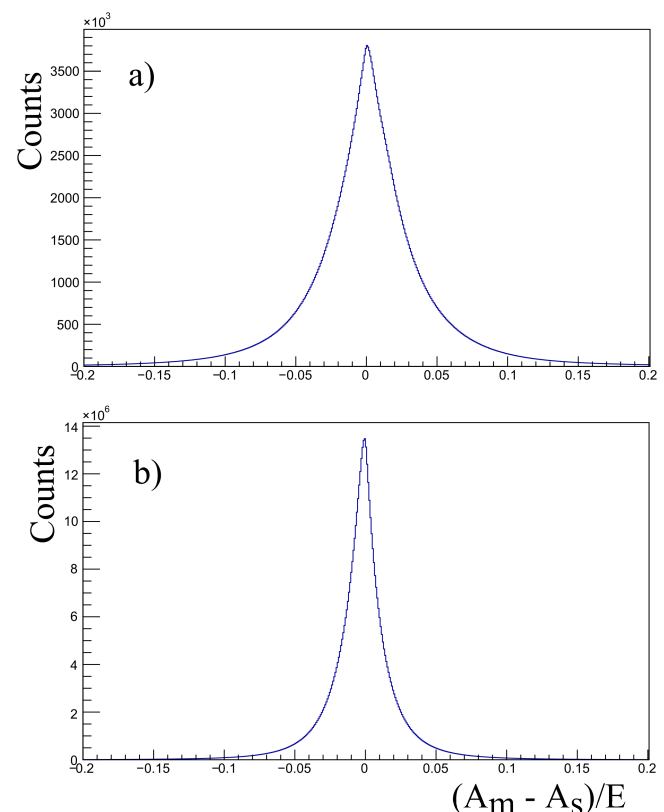

Figure 18. The difference of measured $A_{m}$ and simulated $A_{s}$ pulses using the first $400 \mathrm{~ns}$ (corresponding to 40 samples) after the trigger time $T_{0}$ for the a) signals from hit segment and core and b) the transient signals is shown for a multitude of events. Only events with one interaction per crystal were used and the pulses $A_{m}$ and $A_{s}$ were normalized to the energy of the interaction $E$. The resulting distributions are not Gaussian like but have left and right tails.

metric for transient signals on the one hand and for the hit segment and the core on the other hand might improve even more the PSA results. Therefore a refined figure of merit was scrutinized:

$$
\mathrm{FOM}=\underbrace{\sum_{l, t_{i}}\left|A_{l, m}\left(t_{i}\right)-A_{l, s}\left(t_{i}\right)\right|^{q}}_{\text {neighboring segments }}+\underbrace{\sum_{j, t_{i}}\left|A_{j, m}\left(t_{i}\right)-A_{j, s}\left(t_{i}\right)\right|^{p}}_{\text {hit segment and core }}
$$



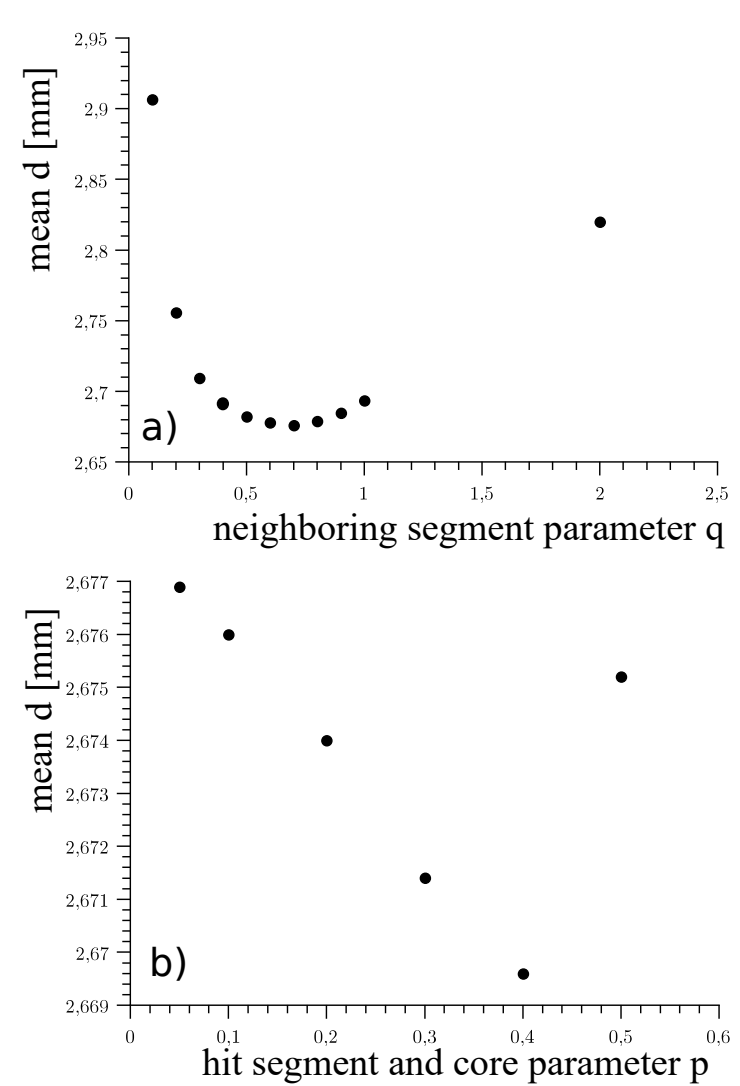

Figure 19. Variation of the new distance metric parameters $p$ and $q$ from eq. 4. An improved PSA performance is achieved by treating the the transient signals individually in the comparison of measurement and simulation.

Two different exponents $q$ and $p$ were evaluated separately in an iterative procedure by leaving one constant and varying the other. The weighting factor for the transient signals was set to 1 for this investigation. Results of the two last iterative steps are shown in Fig. 19. The best PSA result is obtained for $p=0.4$ and $q=0.7$. Note that in the last iteration with a constant $q$ of 0.7 the PSA-performance parameter changes only very slightly in the range from 0.1 to 0.5 . The main improvement is caused by the condition $p<q$. This is most likely caused by the reduced signal to noise ratio in the small transient signals.

\section{Conclusion and outlook}

The results of the AGATA Pulse-Shape Analysis were investigated and improved by exploiting the $180^{\circ} \gamma \gamma \mathrm{co}-$ incidences of annihilated positrons emitted from a ${ }^{22} \mathrm{Na}$ source. An enhanced position resolution was achieved with an adjusted electronic response which is described by a rise time $\tau$ that is $5 \mathrm{~ns}$ larger than previously measured. Simultaneously, the mobility of the holes $\mu$ was reduced by about $10 \%$ to $55 \frac{\mathrm{cm}^{2}}{\mathrm{mVs}}$. A smaller mobility and a slower electronic response have a similar impact on the simulated pulse shapes. Therefore, these two parameters were determined in an iterative procedure. The final mobility is in
Table 2. A summary for the initial and final values of the distance metric parameters $p$ and $q$, the weighting coefficient $w_{j}$, the hole mobility $\mu$ and the preamplifier rise time $\tau$ is shown. The small difference of the mobility $\mu$ in the $\langle 100\rangle$ and $\langle 111\rangle$ directions could not be resolved.

\begin{tabular}{lll}
\hline parameter & initial value & $\begin{array}{l}\text { result after } \\
\text { variation }\end{array}$ \\
\hline$p$ & 0.3 & 0.4 \\
$q$ & 0.3 & 0.7 \\
$w_{j}$ & 1 & 2.75 \\
$\mu_{\langle 100\rangle}$ & $62.934 \frac{\mathrm{cm}^{2}}{\mathrm{mVs}^{2}}$ & $55 \frac{\mathrm{cm}^{2}}{\mathrm{mVs}^{2}}$ \\
$\mu_{\langle 111\rangle}$ & $62.383 \frac{\mathrm{cm}^{2}}{\mathrm{mVs}^{2}}$ & $55 \frac{\mathrm{cm}^{2}}{\mathrm{mVs}^{2}}$ \\
$\tau$ & $35 \mathrm{~ns}$ & $40 \mathrm{~ns}$
\end{tabular}

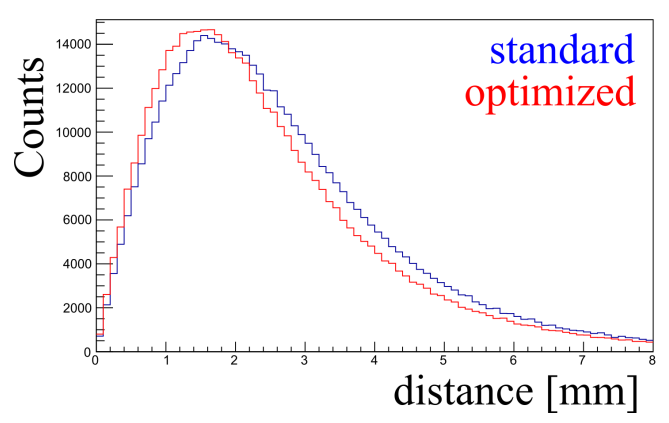

Figure 20. The distribution of distances of the lines connecting the coincident interaction positions to the source position is shown for the initial and optimized set of PSA input parameters.

good agreement with the previously measured value [11]. Remaining differences might originate from the transfer function as well as from the impurity profile of the crystal.

In addition the results of the grid-search algorithm were improved by implementing a weighting factor for the transient signals of the nearest neighbors of the hit segment. A weighting factor of 2.75 yielded the best results. For future investigations it might be worthwhile to introduce an energy dependent weighting factor which increases for higher energies. For low energy depositions the signal to noise ratio is small, especially for the transient signals. In these cases a stronger weighting of these signals might not be favorable. However, a different approach has to be applied in order to determine the optimal energy dependence of weighting factors. Unfortunately, the ${ }^{22} \mathrm{Na}$-coincidence method is restricted to energy depositions of $511 \mathrm{keV}$.

It was shown that an Euclidean metric, corresponding to an exponent value of two in the figure of merit calculation, does not give best PSA results. Improvements were achieved by splitting the figure of merit calculation into two parts for the hit segment and core, as well as for the neighboring segments. The best PSA performance was observed by choosing the exponent to 0.4 for the hit segment and core and to 0.7 for the neighboring segments.

The final improved PSA results are shown in the distribution of interaction positions in Fig. 21 and 22. A clear reduction of the non-physical allocation of hits is obtained: 

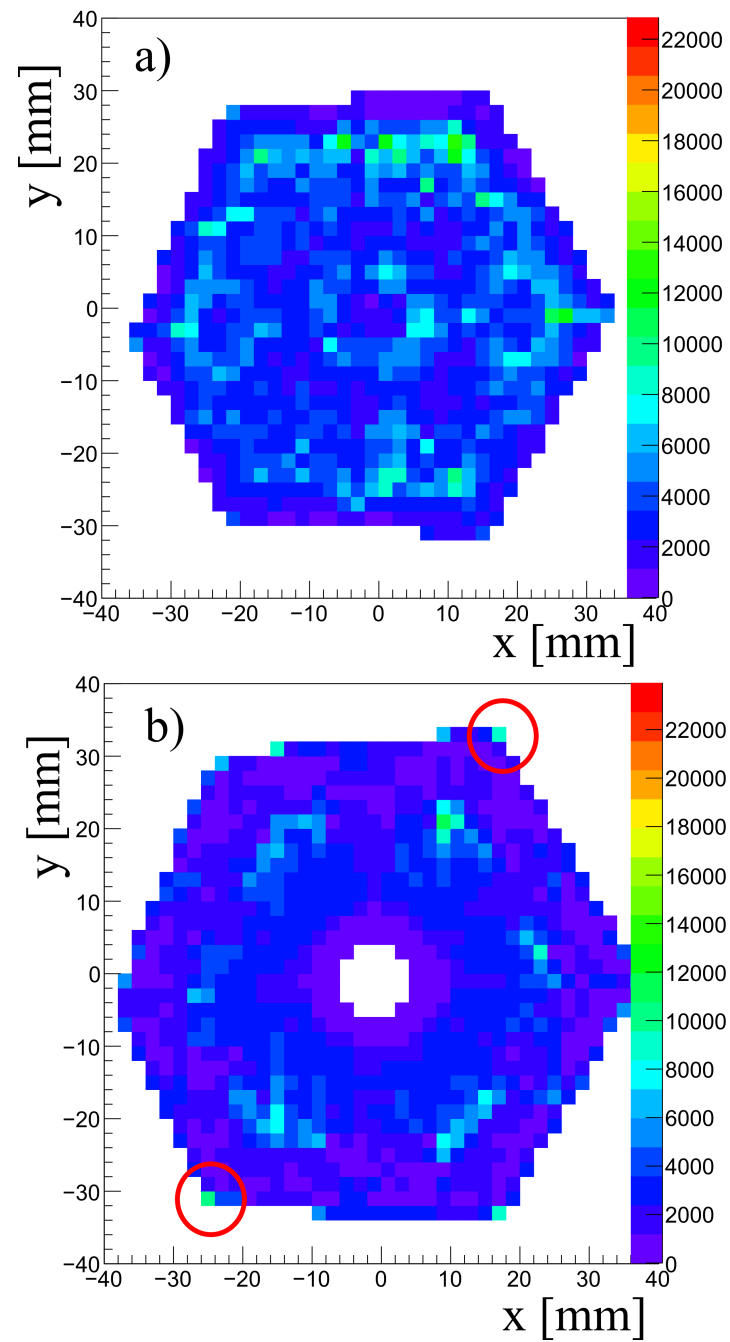

Figure 21. The optimized results of the PSA are shown for the same slices as in Fig. 3. Picture a) shows the slice for $\mathrm{z}=6-8 \mathrm{~mm}$ and $b$ ) for $\mathrm{z}=28-30 \mathrm{~mm}$. The same color code was chosen like in Fig. 3.

The visibility of the segment structure is diminished. The number of hits at large radii is no longer smaller than expected. The amount of interactions in single high statistics grid points is decreased to a more reasonable level. A comparison of the distances of the coincidence lines to the source is shown in Fig. 20. The standard configuration yields a mean distance of $2.73 \mathrm{~mm}$ and a standard deviation of $1.85 \mathrm{~mm}$ whereas in the optimized configuration a mean distance of $2.54 \mathrm{~mm}$ and a standard deviation of $1.80 \mathrm{~mm}$ is obtained.

The presented procedure was able to improve the PSA performance, although remaining limitations have to be accounted for. The choice of the parameters and its variation is motivated by an empiric approach. The starting values were taken from measurements and then varied in the final configuration of the spectrometer and its PSA grid search algorithm. The resulting improved parameters, for example for the transfer function, are the ones that yield the best position resolution, but do not necessarily have to coincide
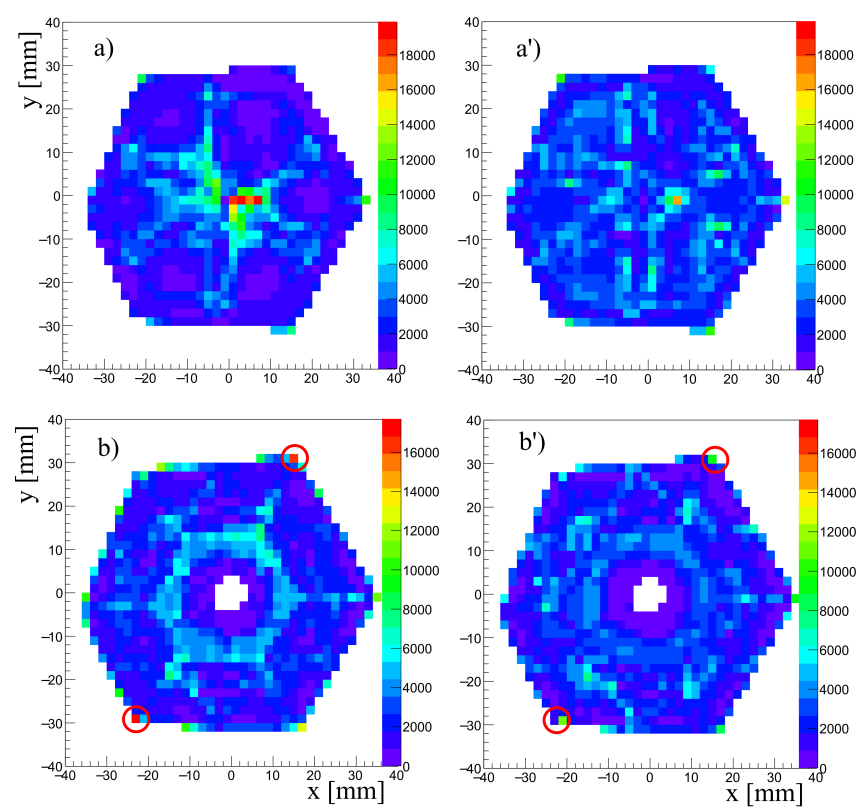

Figure 22. A comparison between initial (left) and optimized (right) PSA results is shown for a) $\mathrm{z}=4-6 \mathrm{~mm}$ and b) $\mathrm{z}=14$ $16 \mathrm{~mm}$. A distinct improvement of the allocated interaction positions is observed. The same color code was chosen for a) and a') and b) and b').

with the physical ones. This is caused by the fact that the PSA performance could only be determined for a subset of input parameters.

In future, a full scan of the N-dimensional performance landscape should be performed and the long processing time of each variation step (where $\mathrm{N}$ is the number of input parameters) has to be coped with. In this way the variation will provide the global optimal configuration in the $\mathrm{N}$ dimensional space. Also the $\gamma$-ray energy should not be restricted to $511 \mathrm{keV}$. Previous investigations showed that PSA performance depends on the energy deposition at the interaction point due to the better signal to noise ratio at higher energies [16]. The restriction to a single energy may produce biased results and a continued effort is needed to resolve the energy dependence of the Pulse-Shape Analysis.

The ${ }^{22} \mathrm{Na}$-coincidence method was established as a fast and reliable tool to study the results of the PSA, improve the simulated signal basis and the position resolution. The electronic response, the hole mobility and features of the grid search algorithm were investigated and adjusted. Despite the improvements made some unexpected distribution of hits, e.g. a clustering of hits, in the detector is still observed. Therefore, a continued investigation is needed to obtain better reference pulses and an improved search algorithm. In particular two interactions inside a single segment should be considered. The current algorithm assumes only single interactions inside a segment. As a result two hits inside a segment are allocated at the energy weighted bary center of the interaction. The disentanglement of one hit and two hit interactions inside a single segment is very 
challenging and requires a high fidelity of the reference basis. Several scanning tables were developed to obtain reference pulses at known interaction positions [17, 18, 19]. In future a detailed comparison with scanning table results should be utilized to simulate an improved signal basis.

This research was supported by the German BMBF under Grants 06K-167, 06KY205I, 05P12PKFNE, 05P15PKFN9, 05P18PKFN9. AGATA was supported by the European funding bodies and the EU Contract RII3-CT-2004-506065.
18. F. Crespi, F. Camera, B. Million, M. Sassi, O. Wieland, A. Bracco, Nucl. Instrum. Methods A 593(3), 440 (2008)

19. A. Hernndez-Prieto, B. Quintana, D. Barrientos, High spatial resolution -camera devoted to characterization of electrical response in HPGe segmented detectors, in 2011 IEEE Nuclear Science Symposium Conference Record (2011), pp. 1506-1509

\section{References}

1. S. Akkoyun et al., Nucl. Instrum. Methods A 668, 26 (2012)

2. A. Lopez-Martens, K. Hauschild, A. Korichi, J. Roccaz, J.P. Thibaud, Nucl. Instrum. Methods A 533(3), 454 (2004)

3. D.B. R. Venturelli, LNL Annual Report 2004 p. 220 (2005)

4. B. Bruyneel, B. Birkenbach, P. Reiter, Eur. Phys. J A 52(3), 1 (2016), ISSN 1434-601X

5. F. Recchia, D. Bazzacco, E. Farnea, A. Gadea, R. Venturelli et al., Nucl. Instrum. Methods A 604(3), 555 (2009)

6. N. Lalovi, C. Louchart, C. Michelagnoli, R. PerezVidal, D. Ralet, J. Gerl, D. Rudolph et al., Nucl. Instrum. Methods A 806, 258 (2016)

7. J. Dryzek, Detection of Positron Implantation Profile in different Materials, in Proceedings of the 35th Polish Seminar on Positron Annihilation (2004)

8. S. Klupp, Master's thesis, Technische Universität München (2011)

9. G. Pascovici, A. Pullia, F. Zocca, B. Bruyneel, D. Bazzacco, WSEAS Trans. Cir. and Sys. 7(6), 470 (2008)

10. A. Pullia, G. Pascovici, B. Cahan, D.E. Weisshaar, C. Boiano, R. Bassini, M. Petcu, F. Zocca, IEEE Symposium Conference Record Nuclear Science 2004. 3, 1411 (2004)

11. B. Bruyneel, P. Reiter, G. Pascovici, Nucl. Instrum. Methods A 569(3), 774 (2006)

12. B. Bruyneel, P. Reiter, G. Pascovici, Nucl. Instrum. Methods A 569(3), 764 (2006)

13. B. Bruyneel, B. Birkenbach, P. Reiter, Eur. Phys. J. A 52(3), 70 (2016)

14. V. Prasher, M. Cromaz, E. Merchan, P. Chowdhury, H. Crawford, C. Lister, C. Campbell, I. Lee, A. Macchiavelli, D. Radford et al., Nucl. Instrum. Methods A 846(Supplement C), 50 (2017)

15. F. Recchia, Ph.D. thesis, Universita degli Studi di Padova (2008)

16. P.A. Söderström, F. Recchia, J. Nyberg et al., Nucl. Instrum. Methods A 638(1), 96 (2011)

17. T. Ha, A. Korichi, F.L. Blanc, P. Dsesquelles, N. Dosme, X. Grave, N. Karkour, S. Leboutelier, E. Legay, D. Linget et al., Nucl. Instrum. Methods A 697, 123 (2013) 